\title{
METAPHYSICS IN KÖNIGSBERG \\ PRIOR TO KANT (1703-1770)
}

Marco SGARBI*

\begin{abstract}
A BSTRACT: The present contribute aims to reconstruct, using the methodology of intellectual history, the broad spectrum of metaphysical doctrines that Kant could know during the years of the formation of his philosophy. The first part deals with the teaching of metaphysics in Königsberg from 1703 to 1770. The second part examines the main characteristics of the metaphysics in the various handbooks, which were taught at the Albertina, in order to have an exhaustive overview of all metaphysical positions.
\end{abstract}

KE YWORDS: Metaphysics. Eclecticism. Wolffianism. A ristotelianism. Kant. Königsberg. Quellengeschichte.

\section{Introduction}

The Kant-Forschung has never paid a lot of attention to the reconstruction of the Kantian philosophy beginning from the cultural background of Königsberg's university. Working on Königsberg's framew ork

\footnotetext{
* Marco Sgarbi studies Kantian philosophy, German Enlightenment, and the history of Aristotelian tradition. He has published the following books: La Kritik der reinen Vernunft nel contesto della tradizione logica aristotelica (Olms, 2010) Logica e metafisica nel Kant precritico. L'ambiente intellettuale di Königsberg e la formazione della filosofia kantiana (Peter Lang, 2010); La logica dell'irrazionale. Studio sul significato e sui problemi della Kritik der Urteilskraft (M imesis, 2010). He has published more than ten volumes and he has published in «Rivista di Storia della Filosofia», «Rivista di Filosofia Neo-scolastica», «M edioevo», «A rchiv für Begriffsgeschichte», and «Fenomenologia e Società».
} 
does not mean to deal with Kant's biography, but to understand if and how the cultural context, in which he grew up, had influenced his philosophical perspectives particularly in the metaphysical field. In fact, according to Giorgio Tonelli, who wrote the only meaningful investigation in this sense (TONELLI, 1975), the A lbertina was a battlefield between philosophical and religious positions that determined decisevly Kant's development. Tonelli, unfortunately, had no the Vorlesungsverzeichnisse available, which have been rediscovered by Riccardo Pozzo two decades ago and which shed light upon the conditions in Königsberg and the making of Kant's philosophy in three crucial moments: 1) from 1703 to 1740 , before Kant's matriculation; 2) from 1740 to 1746 , when Kant was a university student; 3) from 1746 to 1770, when he was lecturer at the Albertina.

The present contribute aims to reconstruct the broad spectrum of metaphysical doctrines that Kant could know during the years of the formation of his philosophy.

The first part, following the methodology of the new edition of the Grundriss der Geschichte der Philosophie and of the Quellengeschichte, ${ }^{1}$ deals with the teaching of metaphysics in Königsberg from 1703 to 1770, using the unpublished materials of the Ratio praelectionum (1703-1719). ${ }^{2}$ The second part examines the definition, the subject, and the divison of metaphysics in the various handbooks, which were taught at the Albertina, in order to have an exhaustive overview of all metaphysical positions.

\section{The teaching of metaphysics in Königsberg}

A fruitful approach to study metaphysics in Königsberg between 1703 to 1770 is to reconstruct the history of the chair of "logic and metaphysics". The chair was founded in 1552 and it was simply of "dialectics", as it was usual in that epoch when metaphysics was incorporated into logic and the metaphysical problems were investigated in theology (POZZO, 2004). During the $17^{\text {th }}$ century the chair became of "logic" and then of "logic and metaphysics". From its foundation the chair was occupied by Nikolaus J agenteufel (1552-1567), M artin Lauben (1569-1578), Michael Scrinius (1579-

\footnotetext{
${ }^{1}$ The Quellengeschichte focuses its attention on six issues to reconstruct Kantian philosophy: 1) the statement of Kant; 2) the references in the Briefwechsel and in the Nachlass; 3) the references in other authors; 4) university professors; 5) the textbooks at the Albertina; 6) the intellectual background of Königsberg (HINSKE, 2006). This paper deals particularly with the last three issues.
}

2 I am preparing a critical edition of Königsberg's Ratio praelectionum (1703-1719). 
1585), Lorenz Pantän (1585-1589), M artin Winter (1589-1595), J ohann Geldern (1595-1620), Georg Crusius (1621-1625), Levin Pouchen (1626), Lorenz Weger (1626-1629), Micheal Eifler (1630-1657), Melchior Zeidler (1658-1663), Lambert Steger (1663-1667), and Andreas Hedio (1667-1703) (PISANSKI, 1886, p. 149, 291).

Many of these professors were extremely important for the history of Königsberg's university and for the development of the Schulphilosophie in Germany. For example Crusius was the first to introduce the Suárez's metaphysics at the A lbertina, Calov invented the new sciences of gnostologia, noologia, and methodologia, contributing also to the development of the ontology, Zeidler imported J acopo Zabarella's methodological works and Hedio w rote the most exhaustive and exstensive commentary to A ristotle's Organon of the $17^{\text {th }}$ century. These few examples show how much the chair was charaterized by A ristotelianism and Scholastic philosophy.

This is the framework within it is necessary to contextualize the first professor of logic and metaphysics of the $18^{\text {th }}$ century: Paul Rabe (SGARBI, 2009a). Rabe was professor of logic and metaphysics from 1703 until his death in 1713. His main contribute in the field of metaphysics is Cursus philosophicus, seu Compendium praecipuarum scientiarum philosophicarum, Dialecticae nempe, Analyticae, Politicae, sub qua comprehenditur Ethica, Physicae atque M etaphysicae. After Rabe, J ohann Böse was professor of logic and metaphysics from 1713 to 1719, but he never wrote on metaphysical topics. From 1715 to 1725 Heinrich Oelmann was associate professor of logic and metaphysics. He is important because was the first to lecture Wolffian metaphysics in Königsberg. After Rabe, the most important A ristotelian was J ohann J akob Rohde, who was professor of logic and metaphysics from 1720 to 1727 . He was A ristotelian during all his life, teaching A ristotelian metaphysics, as the academic program M editatione philosophica qua A ristotelica sapientissimus de veritate judice shows (ROHDE, 1722). A nother important A ristotelian and a pupil of Rabe was J ohann David Kypke, who had the chair of logic and metaphysics from 1725 to 1758 , and also hosted Kant for a while in his house. ${ }^{3}$ From 1728 to 1729 the A ristotelian J ohann Gottfried Teske, who was extremley close to Kant, became associate professor of logic and metaphysics. After Teske, the future theologician Daniel Lorenz Salthenius was associate professor of logic and metaphysics between 1729 and 1732. He was usual to teach and comment the A ristotelian w orks during his lectures. From 1732 to 1733J ohann Georg Bock, one of the most important aestheticians of his time, had the chair

${ }^{3}$ Records are extremely ambiguous, and some believe he lodged with Kypke's nephew, J ohann Georg. Anyway the important thing is that Kant was familiar with Kypke's house. 
of logic and metaphysics, which was subsequently occupied in 1733 by the eclectic Konrad Gottlieb M arquardt, who was Kant's professor of mathematics. ${ }^{4}$ From 1734 to 1751 Martin Knutzen, who was Kant's mentor, became associate professor of logic and metaphysics. A fter Kypke's death, Friedrich J ohann Buck became from 1759 to 1770 had the chair until Kant replaced him.

For an exhaustive investigation on Königsberg's metaphysical background it is necessary to consider also the teachings at the Albertina in order to understand the main trends and influences of the period and the various changes during times.

Before to analyze the teaching of metaphysics in each semester, it is important to recall that in Königsberg in order to be admitted to the faculty of theology it was required to complete the whole cursus philosophicus, which was composed by six different philosophical disciplines: 1) dialectics; 2) analytics; 3) ethics; 4) politics; 5) physics; 6) metaphysics (ERDM AN N, 1876, p. 21). This cursus could be taught on different handbooks (POZZO, 1991, p. 75), even if the more suitable handbook for this kind of teaching was Rabe's Cursus philosophicus (SGARBI, 2009a, p. 276-278). In fact, it was highly recommended to the students of theology, law, and medicine as its subtitle says explicitly - "in superioribus Facultatibus usui esse potest in Theologia nempe, J urisprudentia et M edicina" - and it was divided in dialectics, analytics, ethics, politics, natural philosophy and metaphysics. The fortune of this handbook is testified by the publication in 1716 of its companion Philosophia propaedeutica sive Philosophiae Fundamenta praerequisita, ad ductum et methodum Cursus Philosophici b. Professoris Raben in tres tomos iuxta triplicem Philosophiam breviter et perspicue distributa (PISANSKI, 1886, p. 529). It is reasonable to suppose that Rabe's Cursus was for its propaedeuticity and ufficiality the reference handbook of the cursus philosophicus. Other possible handbooks to study the cursus were Franz A lbert A epinus's Introductio in philosophiam, Ludwig Thümmig's Institutiones philosophiae wolfianae, J ohann Georg Walch's Introductio in Philosophiam, J ohann Christoph Gottsched's Erste Gründe der gesammten Weltweisheit, Christian Friedrich Ammon's Lineae primae eruditionis humanae. Only occasionally the cursus philosophicus was thaught on the five Wolffian manuals: Vernünftige Gedanken von den Kräfften des meschlichen Verstandes und ihrem richtigen Gebrauch in Erkäntniß der Wahrheit; Vernünftige Gedanken von Gott, der Welt und der Seele des M enschen, auch allen Dingen überhaupt; Vernünftige Gedanken von der M enschen Thun und Lassen; Vernünftige Gedanken von

\footnotetext{
${ }^{4}$ Pisanski characterizes Marquardt as an Aristotelian, while Tonelli and Rumore as a Wolffian. Probably he was an Eclectic (PISANSKI, 1886, p. 532).
} 
dem gesellschafftlichen Leben der $M$ enschen und insonderheit dem gemeinen Wesen; Vernünftige Gedanken von der Würckungen der Natur. All these handbooks, however, did not encompass all the cursus philosophicus as the Rabe's Cursus did.

\section{Lectures on Metaphysics (1703-1770)}

(ns) $=$ the handbook for the lectures on metaphysics is not specified.

$(\mathrm{cp})=$ cursus philosophicus, when there is also (ns) or a handbook, it means that besides the teaching of metaphysics, it was taught also the cursus philosophicus, which could include also lectures on metaphysics.

$(p)=$ probable, when it is not specified but it is possible to deduce it from the Vorlesungsverzeichnisse or from previous lectures.

\begin{tabular}{|l|l|}
\hline Professors & Winter Semester 1703/1704 \\
\hline Paul Rabe & Abraham Calov, Metaphysica divina, Hallervord, Rostock 1640. (p) \\
\hline
\end{tabular}

\begin{tabular}{|l|c|}
\hline Professors & Summer Semester 1704 \\
\hline Paul Rabe & Paul Rabe, Cursus philosophicus, Boye, Königsberg 1703. (c) \\
\hline
\end{tabular}

\begin{tabular}{|l|l|}
\hline Professors & Winter Semester 1704/1705 \\
\hline Paul Rabe & Paul Rabe, Cursus philosophicus, Boye, Königsberg 1703. (c) \\
\hline
\end{tabular}

\begin{tabular}{|l|l|}
\hline Professors & Summer Semester 1704 \\
\hline Paul Rabe & Paul Rabe, Cursus philosophicus, Boye, Königsberg 1703. (c) \\
\hline
\end{tabular}

\begin{tabular}{|l|l|}
\hline Professors & Summer Semester 1705 \\
\hline Paul Rabe & Paul Rabe, Cursus philosophicus, Boye, Königsberg 1703. (c) \\
\hline
\end{tabular}

\begin{tabular}{|l|l|}
\hline Professors & Winter Semester 1705/1706 \\
\hline Paul Rabe & Paul Rabe, Cursus philosophicus, Boye, Königsberg 1703. (c) \\
\hline
\end{tabular}

\begin{tabular}{|c|}
\hline Summer Semester 1706 \\
\hline There were not lectures on metaphysics in this semester \\
\hline
\end{tabular}

\section{Winter Semester 1706/1707}

There were not lectures on metaphysics in this semester 


\begin{tabular}{|l|l|}
\hline Professors & Summer Semester 1707 \\
\hline Paul Rabe & Paul Rabe, Cursus philosophicus, Boye, Königsberg 1703. (c) \\
\hline
\end{tabular}

\begin{tabular}{|l|l|}
\hline Professors & Winter Semester 1707/1708 \\
\hline Paul Rabe & Paul Rabe, Cursus philosophicus, Boye, Königsberg 1703. (c) \\
\hline
\end{tabular}

\begin{tabular}{|l|l|}
\hline Professors & Summer Semester 1708 \\
\hline Paul Rabe & Paul Rabe, Cursus philosophicus, Boye, Königsberg 1703. (c) \\
\hline
\end{tabular}

\begin{tabular}{|l|l|}
\hline Professors & Winter Semester 1708/1709 \\
\hline Paul Rabe & Paul Rabe, Cursus philosophicus, Boye, Königsberg 1703. (c) \\
\hline
\end{tabular}

\begin{tabular}{|l|l|}
\hline Professors & Summer Semester 1709 \\
\hline Paul Rabe & Paul Rabe, Cursus philosophicus, Boye, Königsberg 1703. (c) \\
\hline
\end{tabular}

\begin{tabular}{|l|l|}
\hline Professors & Winter Semester 1709/1710 \\
\hline Paul Rabe & Paul Rabe, Cursus philosophicus, Boye, Königsberg 1703. (c) \\
\hline
\end{tabular}

\begin{tabular}{|l|l|}
\hline Professors & Summer Semester 1710 \\
\hline Paul Rabe & Paul Rabe, Cursus philosophicus, Boye, Königsberg 1703. (c) \\
\hline
\end{tabular}

\begin{tabular}{|l|l|}
\hline Professors & Winter Semester 1710/1711 \\
\hline Paul Rabe & Paul Rabe, Cursus philosophicus, Boye, Königsberg 1703. (c) \\
\hline
\end{tabular}

\begin{tabular}{|l|l|}
\hline Professors & Summer Semester 1711 \\
\hline Paul Rabe & Paul Rabe, Cursus philosophicus, Boye, Königsberg 1703. (c) \\
\hline
\end{tabular}

\begin{tabular}{|l|l|}
\hline Professors & Winter Semester 1711/1712 \\
\hline Paul Rabe & Paul Rabe, Cursus philosophicus, Boye, Königsberg 1703. (c) \\
\hline
\end{tabular}

\begin{tabular}{|l|l|}
\hline Professors & Summer Semester 1712 \\
\hline Paul Rabe & Paul Rabe, Cursus philosophicus, Boye, Königsberg 1703. (c) \\
\hline
\end{tabular}

\begin{tabular}{|l|l|}
\hline Professors & Winter Semester 1712/1713 \\
\hline Paul Rabe & Paul Rabe, Cursus philosophicus, Boye, Königsberg 1703. (c) \\
\hline
\end{tabular}




\begin{tabular}{|l|l|}
\hline Professors & Summer Semester 1713 \\
\hline Paul Rabe & Paul Rabe, Cursus philosophicus, Boye, Königsberg 1703. (c) \\
\hline
\end{tabular}

\begin{tabular}{|c|c|}
\hline Professors & Winter Semester 1713/1714 \\
\hline J ohann Böse & Paul Rabe, Cursus philosophicus, Boye, Königsberg 1703. (c) \\
\hline
\end{tabular}

\begin{tabular}{|c|c|}
\hline Professors & Summer Semester 1714 \\
\hline J ohann Böse & Paul Rabe, Cursus philosophicus, Boye, Königsberg 1703. (c) \\
\hline
\end{tabular}

\begin{tabular}{|c|c|}
\hline Professors & Winter Semester 1714/1715 \\
\hline J ohann Böse & Paul Rabe, Cursus philosophicus, Boye, Königsberg 1703. (c) \\
\hline
\end{tabular}

\begin{tabular}{|c|c|}
\hline Professors & \multicolumn{1}{|c|}{ Summer Semester 1715 } \\
\hline Hieronymus Georgi & Paul Rabe, Cursus philosophicus, Boye, Königsberg 1703. (p) \\
\hline Heinrich Oelmann & Paul Rabe, Cursus philosophicus, Boye, Königsberg 1703. (p) \\
\hline
\end{tabular}

\begin{tabular}{|c|l|}
\hline \multicolumn{1}{|c|}{ Professors } & \multicolumn{1}{|c|}{ Winter Semester 1715/1716 } \\
\hline Hieronymus Georgi & $\begin{array}{l}\text { Hieronymus Georgi, Philosophia propaedeutica sive Philosophiae } \\
\text { Fundamenta praerequisita, ad ductum et methodum Cursus Philosophici } \\
\text { b. Professoris Raben in tres tomos iuxta triplicem Philosophiam breviter et } \\
\text { perspicue distributa, Georgi, Königsberg 1716. }\end{array}$ \\
\hline Heinrich Oelmann & Paul Rabe, Cursus philosophicus, Boye, Königsberg 1703. (p) \\
\hline
\end{tabular}

\begin{tabular}{|c|c|}
\hline Professors & Summer Semester 1716 \\
\hline Heinrich Oelmann & Paul Rabe, Cursus philosophicus, Boye, Königsberg 1703. (p) \\
\hline Micheal Gehrke & Paul Rabe, Cursus philosophicus, Boye, Königsberg 1703. (p) \\
\hline
\end{tabular}

\begin{tabular}{|c|l|}
\hline Professors & \multicolumn{1}{|c|}{ Winter Semester 1716/1717 } \\
\hline Hieronymus Georgi & Paul Rabe, Cursus philosophicus, Boye, Königsberg 1703. (p) \\
\hline Heinrich Oelmann & Abraham Calov, M etaphysica divina, Hallervord, Rostock 1640. \\
\hline Micheal Gehrke & Paul Rabe, Cursus philosophicus, Boye, Königsberg 1703. (p) \\
\hline
\end{tabular}

\section{Summer Semester 1717}

There were not lectures on metaphysics in this semester 


\begin{tabular}{|c|c|}
\hline Professors & \multicolumn{1}{|c|}{ Winter Semester 1717/1718 } \\
\hline Micheal Gehrke & Paul Rabe, Cursus philosophicus, Boye, Königsberg 1703. (p) \\
\hline Heinrich Oelmann & Paul Rabe, Cursus philosophicus, Boye, Königsberg 1703. (p) \\
\hline
\end{tabular}

\begin{tabular}{|c|c|}
\hline Professors & Summer Semester 1718 \\
\hline Heinrich Oelmann & Paul Rabe, Cursus philosophicus, Boye, Königsberg 1703. (p) \\
\hline
\end{tabular}

\begin{tabular}{|c|c|}
\hline Professors & Winter Semester 1718/1719 \\
\hline Micheal Gehrke & Paul Rabe, Cursus philosophicus, Boye, Königsberg 1703. (p) \\
\hline
\end{tabular}

\begin{tabular}{|c|c|}
\hline Professors & Summer Semester 1719 \\
\hline Heinrich Oelmann & Paul Rabe, Cursus philosophicus, Boye, Königsberg 1703. (p) \\
\hline
\end{tabular}

\begin{tabular}{|c|c|}
\hline Professors & Winter Semester 1719/1720 \\
\hline Micheal Gehrke & Paul Rabe, Cursus philosophicus, Boye, Königsberg 1703. (p) \\
\hline
\end{tabular}

\begin{tabular}{|c|l|}
\hline Professors & \multicolumn{1}{|c|}{ Summer Semester 1720 } \\
\hline J ohann J akob Rohde & $\begin{array}{l}\text { J ohann Franz Budde, Elementa philosophiae theoreticae, Orphanotrophii, } \\
\text { Halle 1703. (p) }\end{array}$ \\
\hline Heinrich Oelmann & Paul Rabe, Cursus philosophicus, Boye, Königsberg 1703. (p) \\
\hline Micheal Gehrke & Paul Rabe, Cursus philosophicus, Boye, Königsberg 1703. (p) \\
\hline Georg Heinrich Rast & (ns), (cp) \\
\hline
\end{tabular}

\begin{tabular}{|c|l|}
\hline Professors & \multicolumn{1}{|c|}{ Winter Semester 1720/1721 } \\
\hline J ohann J akob Rohde & $\begin{array}{l}\text { Friedrich Gentzken, Doctrina univeralium quam vocant Metaphysicam, } \\
\text { Reumann, Kilon 1720. }\end{array}$ \\
\hline $\begin{array}{c}\text { Christian Gabriel } \\
\text { Fischer }\end{array}$ & $\begin{array}{l}\text { J ohann Franz Budde, Elementa philosophiae theoreticae, Orphanotrophii, } \\
\text { Halle 1703. }\end{array}$ \\
\hline Micheal Gehrke & Paul Rabe, Cursus philosophicus, Boye, Königsberg 1703. (p) \\
\hline Heinrich Oelmann & $\begin{array}{l}\text { J ohann Franz Budde, Elementa philosophiae theoreticae, Orphanotrophii, } \\
\text { Halle 1703. }\end{array}$ \\
\hline Georg Heinrich Rast & (cp) \\
\hline
\end{tabular}




\begin{tabular}{|c|l|}
\hline Professors & \multicolumn{1}{|c|}{ Summer Semester 1721 } \\
\hline J ohann Jakob Rohde & $\begin{array}{l}\text { Christian Thomasius, Introductio in philosophiam aulicam, Thomasius, } \\
\text { Leipzig 1688. } \\
\text { J ohann Franz Budde, Elementa philosophiae theoreticae, Orphanotrophii, } \\
\text { Halle 1703. }\end{array}$ \\
\hline Heinrich Oelmann & $\begin{array}{l}\text { Christian Wolff, Vernünftige Gedanken von Gott, der Welt und der Seele } \\
\text { des Menschen, auch allen Dingen überhaupt, Renger, Halle 1719. }\end{array}$ \\
\hline
\end{tabular}

\begin{tabular}{|c|l|}
\hline Professors & \multicolumn{1}{|c|}{ Winter Semester 1721/1722 } \\
\hline J ohann J akob Rohde & \multicolumn{1}{|c|}{$(\mathrm{ns})^{5}$} \\
\hline Heinrich Oelmann & $\begin{array}{l}\text { Christian Thomasius, Einleitung zur Hoff-Philosphie, Bauren, Frankfurt } \\
1710 .\end{array}$ \\
\hline
\end{tabular}

\begin{tabular}{|c|l|}
\hline Professors & \multicolumn{1}{|c|}{ Summer Semester 1722 } \\
\hline Heinrich Oelmann & Christian Thomasius, Einleitung zur Hoff-Philosphie, Bauren, Frankfurt \\
& $\begin{array}{l}\text { Christian Wolff, Vernünftige Gedanken von Gott, der Welt und der Seele } \\
\text { des Menschen, auch allen Dingen überhaupt, Renger, Halle 1719. } \\
\end{array}$ \\
& (сp) \\
\hline
\end{tabular}

\begin{tabular}{|c|l|}
\hline Professors & \multicolumn{1}{|c|}{ Winter Semester 1722/1723 } \\
\hline Heinrich Oelmann & $\begin{array}{l}\text { Christian Wolff, Vernünftige Gedanken von Gott, der Welt und der Seele } \\
\text { des Menschen, auch allen Dingen überhaupt, Renger, Halle 1719. }\end{array}$ \\
\hline
\end{tabular}

\begin{tabular}{|c|c|}
\hline Professors & \multicolumn{1}{|c|}{ Summer Semester 1723 } \\
\hline J ohann J akob Rohde & \multicolumn{1}{c|}{ (ns) } \\
\hline Heinrich Oelmann & $\begin{array}{l}\text { Christian Wolff, Vernünftige Gedanken von Gott, der Welt und der Seele } \\
\text { des Menschen, auch allen Dingen überhaupt, Renger, Halle } 1719 .\end{array}$ \\
\hline
\end{tabular}

${ }^{5}$ Rohde taught beginning from February 1722 A ristotelian metaphysics as his Einladungsschrift shows (ROHDE, 1722). 


\begin{tabular}{|c|l|}
\hline Professors & \multicolumn{1}{|c|}{ Winter Semester 1723/1724 } \\
\hline J ohann Jakob Rohde & $\begin{array}{l}\text { J ohann Franz Budde, Theses theologicae de atheismo et superstitione, } \\
\text { Bielck, J ena 1717. }\end{array}$ \\
\hline Heinrich Oelmann & $\begin{array}{l}\text { Christian Wolff, Vernünftige Gedanken von Gott, der Welt und der Seele } \\
\text { des M enschen, auch allen Dingen überhaupt, Renger, Halle 1719. } \\
\text { Paul Rabe, Cursus philosophicus, Boye, Königsberg 1703. (p) }\end{array}$ \\
\hline
\end{tabular}

\begin{tabular}{|c|l|}
\hline \multicolumn{1}{|c|}{ Professors } & \multicolumn{1}{|c|}{ Summer Semester 1724 } \\
\hline J ohann J akob Rohde & $\begin{array}{l}\text { J ohann Franz Budde, Theses theologicae de atheismo et superstitione, } \\
\text { Bielck, J ena 1717. }\end{array}$ \\
\hline Heinrich Oelmann & $\begin{array}{l}\text { Christian Wolf, Vernünftige Gedanken von Gott, der Welt und der Seele } \\
\text { des M enschen, auch allen Dingen überhaupt, Renger, Halle 1719. } \\
\text { Paul Rabe, Cursus philosophicus, Boye, Königsberg 1703. (p) }\end{array}$ \\
\hline Thomas Burckhard & $\begin{array}{l}\text { Franz Albert Aepinus, Metaphysicae ad theologiam applicata, Garmann, } \\
\text { Rostock 1710. }\end{array}$ \\
\hline
\end{tabular}

\begin{tabular}{|c|l|}
\hline \multicolumn{1}{|c|}{ Professors } & \multicolumn{1}{|c|}{ Winter Semester 1724/1725 } \\
\hline Heinrich Oelmann & $\begin{array}{l}\text { Christian Wolff, Vernünftige Gedanken von Gott, der Welt und der Seele } \\
\text { des Menschen, auch allen Dingen überhaupt, Renger, Halle 1719. } \\
\text { Paul Rabe, Cursus philosophicus, Boye, Königsberg 1703. (p) }\end{array}$ \\
\hline Thomas Burckhard & $\begin{array}{l}\text { Franz Albert Aepinus, Metaphysicae ad theologiam applicata, Garmann, } \\
\text { Rostock 1710. }\end{array}$ \\
\hline
\end{tabular}

\begin{tabular}{|c|c|}
\hline Professors & Summer Semester 1725 \\
\hline J ohann Jakob Rohde & Abraham Calov, Scripta philosophica, Wilden, Lübeck 1651. (p) \\
\hline
\end{tabular}

\begin{tabular}{|c|l|}
\hline Professors & \multicolumn{1}{|c|}{ Winter Semester 1725/1726 } \\
\hline J ohann J akob Rohde & A braham Calov, Scripta philosophica, Wilden, Lübeck 1651. (p) \\
\hline Thomas Burckhard & Franz Albert A epinus, Introductio in philosophiam, Wilde, Rostock 1714. \\
\hline
\end{tabular}

\begin{tabular}{|c|c|}
\hline Professors & Summer Semester 1726 \\
\hline J ohann David Kypke & Paul Rabe, Cursus philosophicus, Boye, Königsberg 1703. (p) \\
\hline
\end{tabular}




\begin{tabular}{|c|l|}
\hline Professors & \multicolumn{1}{|c|}{ Winter Semester 1726/1727 } \\
\hline Thomas Burckhard & Franz Albert Aepinus, Introductio in philosophiam, Wilde, Rostock 1714. \\
\hline $\begin{array}{c}\text { Georg Friedrich } \\
\text { Rogall }\end{array}$ & $\begin{array}{l}\text { Christian Wolff, Vernünftige Gedanken von Gott, der Welt und der Seele } \\
\text { des M enschen, auch allen Dingen überhaupt, Renger, Halle } 1719 .\end{array}$ \\
\hline
\end{tabular}

\begin{tabular}{|c|l|}
\hline Professors & \multicolumn{1}{|c|}{ Summer Semester 1727 } \\
\hline Thomas Burckhard & $\begin{array}{l}\text { Franz Albert A epinus, Introductio in philosophiam, Wilde, Rostock 1714. } \\
(\mathrm{p})\end{array}$ \\
\hline $\begin{array}{c}\text { Georg Friedrich } \\
\text { Rogall }\end{array}$ & $\begin{array}{l}\text { Christian Wolff, Vernünftige Gedanken von Gott, der Welt und der Seele } \\
\text { des M enschen, auch allen Dingen überhaupt, Renger, Halle 1719. }\end{array}$ \\
\hline J ohann David Kypke & Paul Rabe, Cursus philosophicus, Boye, Königsberg 1703. (p) \\
\hline
\end{tabular}

\begin{tabular}{|c|l|}
\hline \multicolumn{1}{|c|}{ Professors } & \multicolumn{1}{|c|}{ Winter Semester 1727/1728 } \\
\hline Thomas Burckhard & $\begin{array}{l}\text { Franz Albert Aepinus, Introductio in philosophiam, Wilde, Rostock } 1714 . \\
\text { (p) }\end{array}$ \\
\hline
\end{tabular}

\begin{tabular}{|c|l|}
\hline \multicolumn{1}{|c|}{ Professors } & \multicolumn{1}{|c|}{ Summer Semester 1728 } \\
\hline Thomas Burckhard & $\begin{array}{l}\text { Franz A lbert A epinus, Introductio in philosophiam, Wilde, Rostock } 1714 . \\
\text { (p) }\end{array}$ \\
\hline
\end{tabular}

Winter Semester 1728/1729

There were not lectures on metaphysics in this semester

\begin{tabular}{|c|c|}
\hline Professors & Summer Semester 1729 \\
\hline J ohann David Kypke & Paul Rabe, Cursus philosophicus, Boye, Königsberg 1703. (p) \\
\hline
\end{tabular}

Winter Semester 1729/1730

There were not lectures on metaphysics in this semester

\begin{tabular}{|c|l|}
\hline Professors & \multicolumn{1}{|c|}{ Summer Semester 1730 } \\
\hline Daniel Salthenius & $\begin{array}{l}\text { Christian Dreier, Sapientia sive philosophia prima, Hendel, Königsberg } \\
\text { 1644. (p) }\end{array}$ \\
\hline
\end{tabular}




\begin{tabular}{|c|c|}
\hline Professors & Winter Semester 1730/1731 \\
\hline J ohann David Kypke & Paul Ra be, Cursus philosophicus, Boye, Königsberg 1703. (p) \\
\hline
\end{tabular}

\begin{tabular}{|c|l|}
\hline Professors & \multicolumn{1}{|c|}{ Summer Semester 1731 } \\
\hline J ohann David Kypke & $\begin{array}{l}\text { Paul Rabe, Cursus philosophicus, Boye, Königsberg 1703. (p) } \\
\text { J ohann Georg Walch, Introductio in Philosophiam, Gleditsch, Leipzig 1730. } \\
\text { J ohann Franz Budde, Elementa philosophiae theoreticae, Orphanotrophii, } \\
\text { Halle 1703. }\end{array}$ \\
\hline
\end{tabular}

\begin{tabular}{|c|c|}
\hline Professors & Winter Semester 1731/1732 \\
\hline $\begin{array}{c}\text { Daniel Heinrich } \\
\text { Arnoldt }\end{array}$ & (ns), (cp) \\
\hline
\end{tabular}

\begin{tabular}{|c|c|}
\hline Professors & Summer Semester 1732 \\
\hline Thomas Burckhard & (cp) \\
\hline Karl Heinrich Rappolt & (ns) \\
\hline
\end{tabular}

\begin{tabular}{|c|c|}
\hline Professors & Winter Semester 1732/1733 \\
\hline Thomas Burckhard & (cp) \\
\hline Daniel Heinrich & (cp) \\
Arnoldt & \\
\hline
\end{tabular}

\begin{tabular}{|c|c|}
\hline Professors & Summer Semester 1733 \\
\hline Daniel Heinrich & (ns) \\
Arnoldt & \\
\hline
\end{tabular}

\begin{tabular}{|c|c|}
\hline Professors & Winter Semester 1733/1734 \\
\hline J ohann David Kypke & (ns) \\
\hline Konrad Gottlieb & (cp) \\
Marquardt & \\
\hline
\end{tabular}




\begin{tabular}{|c|c|}
\hline Professors & Summer Semester 1734 \\
\hline Johann David Kypke & (ns) \\
\hline Konrad Gottlieb & (cp) \\
Marquardt & \\
\hline Martin Knutzen & (cp) \\
\hline
\end{tabular}

\begin{tabular}{|c|c|}
\hline Professors & Winter Semester 1734/1735 \\
\hline Johann David Kypke & (ns) \\
\hline $\begin{array}{c}\text { Konrad Gottlieb } \\
\text { Marquardt }\end{array}$ & (cp) \\
\hline Martin Knutzen & (ns) \\
\hline
\end{tabular}

\begin{tabular}{|c|c|}
\hline Professors & Summer Semester 1735 \\
\hline $\begin{array}{c}\text { Konrad Gottlieb } \\
\text { Marquardt }\end{array}$ & (cp) \\
\hline Martin Knutzen & (cp) \\
\hline
\end{tabular}

\begin{tabular}{|c|l|}
\hline Professors & \multicolumn{1}{|c|}{ Winter Semester 1735/1736 } \\
\hline Thomas Burckhard & $\begin{array}{l}\text { Samuel Christian Hollmann, In universam philosophiam introductio, } \\
\text { Henning, Wittenberg 1734. }\end{array}$ \\
\hline $\begin{array}{c}\text { Konrad Gottlieb } \\
\text { Marquardt }\end{array}$ & (cp) \\
\hline Martin Knutzen & (ns) \\
\hline
\end{tabular}

\begin{tabular}{|c|l|}
\hline Professors & \multicolumn{1}{|c|}{ Summer Semester 1736 } \\
\hline $\begin{array}{c}\text { Karl Andreas } \\
\text { Christiani }\end{array}$ & $\begin{array}{l}\text { Ludwig Thümmig, Institutiones philosophiae w olfianae, Renger, Frankfurt } \\
1725-1726 .\end{array}$ \\
\hline $\begin{array}{c}\text { Konrad Gottlieb } \\
\text { Marquardt }\end{array}$ & $\begin{array}{l}\text { Christian Wolff, Vernünftige Gedanken von Gott, der Welt und der Seele } \\
\text { des M enschen, auch allen Dingen überhaupt, Renger, Halle 1719. }\end{array}$ \\
\hline Martin Knutzen & \multicolumn{1}{c}{ (cp) } \\
\hline
\end{tabular}

\begin{tabular}{|c|c|}
\hline Professors & Winter Semester 1736/1737 \\
\hline Karl Andreas Christiani & (cp) \\
\hline $\begin{array}{c}\text { Konrad Gottlieb } \\
\text { Marquardt }\end{array}$ & (cp) \\
\hline Martin Knutzen & (cp) \\
\hline
\end{tabular}




\begin{tabular}{|c|c|}
\hline Professors & Summer Semester 1737 \\
\hline $\begin{array}{c}\text { Karl Andreas } \\
\text { Christiani }\end{array}$ & (cp) \\
\hline $\begin{array}{c}\text { Konrad Gottlieb } \\
\text { Marquardt }\end{array}$ & (cp) \\
\hline Martin Knutzen & (ср) \\
\hline
\end{tabular}

\begin{tabular}{|c|l|}
\hline Professors & \multicolumn{1}{|c|}{ Winter Semester 1737/1738 } \\
\hline Thomas Burckhard & $\begin{array}{l}\text { J ohann Franz Budde, Elementa philosophiae theoreticae, Orphanotrophii, } \\
\text { Halle 1703. (p) }\end{array}$ \\
\hline Martin Knutzen & (cp) \\
\hline $\begin{array}{c}\text { Konrad Gottlieb } \\
\text { Marquardt }\end{array}$ & (cp) \\
\hline
\end{tabular}

\begin{tabular}{|c|c|}
\hline Professors & Summer Semester 1738 \\
\hline $\begin{array}{c}\text { Konrad Gottlieb } \\
\text { Marquardt }\end{array}$ & (cp) \\
\hline Martin Knutzen & (cp) \\
\hline Karl Andreas & (cp) \\
Christiani & \\
\hline
\end{tabular}

\begin{tabular}{|c|c|}
\hline Professors & Winter Semester 1738/1739 \\
\hline $\begin{array}{c}\text { Karl Andreas } \\
\text { Christiani }\end{array}$ & (cp) \\
\hline $\begin{array}{c}\text { Konrad Gottlieb } \\
\text { Marquardt }\end{array}$ & (cp) \\
\hline Martin Knutzen & (cp) \\
\hline Adam Gregorovious & $\begin{array}{c}\text { The Vorlesungsverzeichnisse lists a book Prolegomena by Andreas Hedio, } \\
\text { which, however, does not exist. }\end{array}$ \\
\hline
\end{tabular}

\begin{tabular}{|c|c|}
\hline Professors & Summer Semester 1739 \\
\hline J ohann David Kypke & (ns) \\
\hline Karl Andreas & (cp) \\
Christiani & \\
\hline $\begin{array}{c}\text { Konrad Gottlieb } \\
\text { Marquardt }\end{array}$ & (cp) \\
\hline Martin Knutzen & (cp) \\
\hline
\end{tabular}




\begin{tabular}{|c|c|}
\hline Professors & \multicolumn{1}{|c|}{ Winter Semester 1739/1740 } \\
\hline Thomas Burckhard & $\begin{array}{l}\text { J ohann Franz Budde, Elementa philosophiae theoreticae, Orphanotrophii, } \\
\text { Halle 1703. (p) }\end{array}$ \\
\hline $\begin{array}{c}\text { Karl Andreas } \\
\text { Christiani }\end{array}$ & (cp) \\
\hline $\begin{array}{c}\text { Konrad Gottlieb } \\
\text { Marquardt }\end{array}$ & (cp) \\
\hline Martin Knutzen & (сp) \\
\hline
\end{tabular}

\begin{tabular}{|c|c|}
\hline Professors & Summer Semester 1740 \\
\hline Karl Andreas & (cp) \\
Christiani & \\
\hline $\begin{array}{c}\text { Konrad Gottlieb } \\
\text { Marquardt }\end{array}$ & (cp) \\
\hline Martin Knutzen & (ns), (cp) \\
\hline
\end{tabular}

\begin{tabular}{|c|c|}
\hline Professors & Winter Semester 1740/1741 \\
\hline Karl Andreas & (сp) \\
Christiani & \\
\hline $\begin{array}{c}\text { Konrad Gottlieb } \\
\text { Marquardt }\end{array}$ & (cp) \\
\hline Martin Knutzen & (сp) \\
\hline
\end{tabular}

\begin{tabular}{|c|l|}
\hline Professors & \multicolumn{1}{|c|}{ Summer Semester 1741 } \\
\hline J ohann David Kypke & $\begin{array}{l}\text { Friedrich Christian Baumeister, Institutiones metaphysicae, Zimmermann, } \\
\text { Wittenberg 1738. }\end{array}$ \\
\hline $\begin{array}{c}\text { Karl Andreas } \\
\text { Christiani }\end{array}$ & (cp) \\
\hline $\begin{array}{c}\text { Konrad Gottlieb } \\
\text { Marquardt }\end{array}$ & (сp) \\
\hline Martin Knutzen & (сp) \\
\hline
\end{tabular}

\begin{tabular}{|c|c|}
\hline Professors & Winter Semester 1741/1742 \\
\hline Karl Andreas & (cp) \\
Christiani & \\
\hline Konrad Gottlieb & (cp) \\
\hline
\end{tabular}




\begin{tabular}{|c|c|}
\hline Professors & \multicolumn{1}{|c|}{ Summer Semester 1742 } \\
\hline J ohann David Kypke & $\begin{array}{l}\text { Friedrich Christian Baumeister, Institutiones metaphysicae, Zimmermann, } \\
\text { Wittenberg 1738. (p) }\end{array}$ \\
\hline $\begin{array}{c}\text { Karl Andreas } \\
\text { Christiani }\end{array}$ & (cp) \\
\hline $\begin{array}{c}\text { Konrad Gottlieb } \\
\text { Marquardt }\end{array}$ & (cp) \\
\hline Martin Knutzen & (cp) \\
\hline
\end{tabular}

\begin{tabular}{|c|c|}
\hline Professors & Winter Semester 1742/1743 \\
\hline Thomas Burckhard & Paul Rabe, Cursus philosophicus, Boye, Königsberg 1703. (p) \\
\hline Karl Andreas & (cp) \\
Christiani & (cp) \\
\hline $\begin{array}{c}\text { Konrad Gottlieb } \\
\text { Marquardt }\end{array}$ & (cp) \\
\hline Martin Knutzen &
\end{tabular}

\begin{tabular}{|c|l|}
\hline Professors & \multicolumn{1}{|c|}{ Summer Semester 1743 } \\
\hline J ohann David Kypke & $\begin{array}{l}\text { Friedrich Christian Baumeister, Institutiones metaphysicae, Zimmermann, } \\
\text { Wittenberg 1738. }\end{array}$ \\
\hline Karl Heinrich Rappolt & $\begin{array}{l}\text { Ludwig Thümmig, Institutiones philosophiae w olfianae, Renger, Frankfurt } \\
1725-1726 .\end{array}$ \\
\hline Martin Knutzen & \multicolumn{1}{c|}{ (cp) } \\
\hline $\begin{array}{c}\text { Karl Andreas } \\
\text { Christiani }\end{array}$ & $\begin{array}{l}\text { Friedrich Christian Baumeister, Institutiones metaphysicae, Zimmermann, } \\
\text { Wittenberg 1738. }\end{array}$ \\
\hline
\end{tabular}

\begin{tabular}{|c|c|}
\hline Professors & Winter Semester 1743/1744 \\
\hline Thomas Burckhard & Paul Rabe, Cursus philosophicus, Boye, Königsberg 1703. (p) \\
\hline Karl Andreas & (cp) \\
Christiani & (cp) \\
\hline $\begin{array}{c}\text { Konrad Gottlieb } \\
\text { Marquardt }\end{array}$ & (cp) \\
\hline Martin Knutzen & \\
\hline
\end{tabular}




\begin{tabular}{|c|l|}
\hline Professors & \multicolumn{1}{|c|}{ Summer Semester 1744 } \\
\hline J ohann David Kypke & $\begin{array}{l}\text { Friedrich Christian Baumeister, Institutiones metaphysicae, Zimmermann, } \\
\text { Wittenberg 1738. }\end{array}$ \\
\hline $\begin{array}{c}\text { Karl Andreas } \\
\text { Christiani }\end{array}$ & (ns) \\
\hline $\begin{array}{c}\text { Konrad Gottlieb } \\
\text { Marquardt }\end{array}$ & (cp) \\
\hline Karl Heinrich Rappolt & (cp) \\
\hline Martin Knutzen & (ns) \\
\hline
\end{tabular}

\begin{tabular}{|c|l|}
\hline Professors & \multicolumn{1}{|c|}{ Winter Semester 1744/1745 } \\
\hline J ohann David Kypke & $\begin{array}{l}\text { Friedrich Christian Baumeister, Institutiones metaphysicae, Zimmermann, } \\
\text { Wittenberg 1738. (p) }\end{array}$ \\
\hline $\begin{array}{c}\text { Karl Andreas } \\
\text { Christiani }\end{array}$ & (cp) \\
\hline $\begin{array}{c}\text { Konrad Gottlieb } \\
\text { Marquardt }\end{array}$ & (сp) \\
\hline Martin Knutzen & (сp) \\
\hline
\end{tabular}

\begin{tabular}{|c|c|}
\hline Professors & Summer Semester 1745 \\
\hline $\begin{array}{c}\text { Karl Andreas } \\
\text { Christiani }\end{array}$ & (cp) \\
\hline $\begin{array}{c}\text { Konrad Gottlieb } \\
\text { Marquardt }\end{array}$ & (cp) \\
\hline Martin Knutzen & (cp) \\
\hline Karl Heinrich Rappolt & (cp) \\
\hline
\end{tabular}

\begin{tabular}{|c|l|}
\hline Professors & \multicolumn{1}{|c|}{ Winter Semester 1745/1746 } \\
\hline J ohann David Kypke & $\begin{array}{l}\text { Friedrich Christian Baumeister, Institutiones metaphysicae, Zimmermann, } \\
\text { Wittenberg 1738. (p) }\end{array}$ \\
\hline $\begin{array}{c}\text { Karl Andreas } \\
\text { Christiani }\end{array}$ & (cp) \\
\hline Martin Knutzen & (сp) \\
\hline
\end{tabular}




\begin{tabular}{|c|c|}
\hline Professors & Summer Semester 1746 \\
\hline $\begin{array}{c}\text { Konrad Gottlieb } \\
\text { Marquardt }\end{array}$ & (cp) \\
\hline Martin Knutzen & (ns) \\
\hline Karl Andreas & (cp) \\
Christiani & \\
\hline
\end{tabular}

\begin{tabular}{|c|c|}
\hline Professors & Winter Semester $1746 / 1747$ \\
\hline Karl Andreas & (ns) (cp) \\
Christiani & (cp) \\
\hline $\begin{array}{c}\text { Konrad Gottlieb } \\
\text { Marquardt }\end{array}$ & (cp) \\
\hline Martin Knutzen & (cp) \\
\hline Karl Heinrich Rappolt & (cp) \\
\hline
\end{tabular}

\begin{tabular}{|c|c|}
\hline Professors & \multicolumn{1}{|c|}{ Summer Semester 1747 } \\
\hline J ohann David Kypke & $\begin{array}{l}\text { Friedrich Christian Baumeister, Institutiones metaphysicae, Zimmermann, } \\
\text { Wittenberg 1738. (p) }\end{array}$ \\
\hline $\begin{array}{c}\text { Karl Andreas } \\
\text { Christiani }\end{array}$ & (ns) \\
\hline $\begin{array}{c}\text { Konrad Gottlieb } \\
\text { Marquardt }\end{array}$ & (cp) \\
\hline Martin Knutzen & (ns), (cp) \\
\hline Karl Heinrich Rappolt & (ns) \\
\hline
\end{tabular}

\begin{tabular}{|c|c|}
\hline Professors & Winter Semester 1747/1748 \\
\hline Karl Andreas & (cp) \\
Christiani & (сp) \\
\hline Konrad Gottlieb & \\
Marquardt & (ср) \\
\hline Martin Knutzen & \\
\hline
\end{tabular}

\begin{tabular}{|c|l|}
\hline Professors & \multicolumn{1}{|c|}{ Summer Semester 1748 } \\
\hline J ohann David Kypke & $\begin{array}{l}\text { Friedrich Christian Baumeister, Institutiones metaphysicae, Zimmermann, } \\
\text { Wittenberg 1738. (p) }\end{array}$ \\
\hline Coelestin Christian & \multicolumn{1}{|c|}{ (ns) } \\
\hline
\end{tabular}




\begin{tabular}{|c|c|}
\hline Professors & Winter Semester 1748/1749 \\
\hline Karl Andreas & (cp) \\
Christiani & \\
\hline $\begin{array}{c}\text { Konrad Gottlieb } \\
\text { Marquardt }\end{array}$ & (cp) \\
\hline Martin Knutzen & (ns) \\
\hline Karl Heinrich Rappolt & (cp) \\
\hline
\end{tabular}

\begin{tabular}{|c|c|}
\hline Professors & \multicolumn{2}{|c|}{ Summer Semester 1749 } \\
\hline Johann David Kypke & $\begin{array}{l}\text { Friedrich Christian Baumeister, Institutiones metaphysicae, Zimmermann, } \\
\text { Wittenberg 1738. (p) }\end{array}$ \\
\hline $\begin{array}{c}\text { Coelestin Christian } \\
\text { Flottwell }\end{array}$ & (ns) \\
\hline Martin Knutzen & (cp) \\
\hline Karl Andreas \\
Christiani
\end{tabular}

\begin{tabular}{|c|l|}
\hline Professors & \multicolumn{1}{|c|}{ Winter Semester 1749/1750 } \\
\hline Martin Knutzen & \multicolumn{1}{c|}{ (сp) } \\
\hline $\begin{array}{c}\text { Coelestin Christian } \\
\text { Flottwell }\end{array}$ & $\begin{array}{l}\text { J ohann Christoph Gottsched, Erste Gründe der gesammten Weltw eisheit, } \\
\text { Breitkopf, Leipzig 1733-1734. }\end{array}$ \\
\hline
\end{tabular}

\begin{tabular}{|c|c|}
\hline Professors & \multicolumn{1}{|c|}{ Summer Semester 1750 } \\
\hline J ohann David Kypke & $\begin{array}{l}\text { Friedrich Christian Baumeister, Institutiones metaphysicae, Zimmermann, } \\
\text { Wittenberg 1738. (p) }\end{array}$ \\
\hline Martin Knutzen & (cp) \\
\hline $\begin{array}{c}\text { Karl Andreas } \\
\text { Christiani }\end{array}$ & (ns), (cp) \\
\hline
\end{tabular}

\begin{tabular}{|c|c|}
\hline Professors & Winter Semester 1750/1751 \\
\hline $\begin{array}{c}\text { Karl Andreas } \\
\text { Christiani }\end{array}$ & \multicolumn{1}{|c|}{ (ns), (cp) } \\
\hline $\begin{array}{c}\text { Coelestin Christian } \\
\text { Flottwell }\end{array}$ & $\begin{array}{l}\text { J ohann Christoph Gottsched, Erste Gründe der gesammten Weltweisheit, } \\
\text { Breitkopf, Leipzig 1733-1734. (p) }\end{array}$ \\
\hline Martin Knuzten & (ns), (cp) \\
\hline
\end{tabular}




\begin{tabular}{|c|l|}
\hline Professors & \multicolumn{1}{|c|}{ Summer Semester 1751 } \\
\hline J ohann David Kypke & $\begin{array}{l}\text { Friedrich Christian Baumeister, Institutiones metaphysicae, Zimmermann, } \\
\text { Wittenberg 1738. }(p)\end{array}$ \\
\hline $\begin{array}{c}\text { Coelestin Christian } \\
\text { Flottwell }\end{array}$ & $\begin{array}{l}\text { J ohann Christoph Gottsched, Erste Gründe der gesammten Weltweisheit, } \\
\text { Breitkopf, Leipzig 1733-1734. }\end{array}$ \\
\hline $\begin{array}{c}\text { Karl Andreas } \\
\text { Christiani }\end{array}$ & \\
\hline
\end{tabular}

\begin{tabular}{|c|l|}
\hline $\begin{array}{c}\text { Professors } \\
\begin{array}{c}\text { Coelestin Christian } \\
\text { Flottwell }\end{array}\end{array}$ & $\begin{array}{c}\text { J } \text { Whanter Semester 1751/1752 } \\
\text { Breitkopf, Leipzig } 1733-1734 .\end{array}$ \\
\hline $\begin{array}{c}\text { Karl Andreas } \\
\text { Christiani }\end{array}$ & (ns) \\
\hline
\end{tabular}

\begin{tabular}{|c|l|}
\hline Professors & \multicolumn{1}{|c|}{ Summer Semester 1752 } \\
\hline J ohann David Kypke & $\begin{array}{l}\text { Friedrich Christian Baumeister, Institutiones metaphysicae, Zimmermann, } \\
\text { Wittenberg 1738. }\end{array}$ \\
\hline $\begin{array}{c}\text { Coelestin Christian } \\
\text { Flottwell }\end{array}$ & (ns) \\
\hline $\begin{array}{c}\text { Karl Andreas } \\
\text { Christiani }\end{array}$ & (ns), (cp) \\
\hline $\begin{array}{c}\text { Henrich Wilhelm } \\
\text { Johanssen }\end{array}$ & (ns) \\
\hline
\end{tabular}

\begin{tabular}{|c|c|}
\hline Professors & Winter Semester 1752/1753 \\
\hline Karl Andreas & (ns), (cp) \\
Christiani & \\
\hline
\end{tabular}

\begin{tabular}{|c|l|}
\hline Professors & \multicolumn{1}{|c|}{ Summer Semester 1753 } \\
\hline J ohann David Kypke & $\begin{array}{l}\text { Friedrich Christian Baumeister, Institutiones metaphysicae, Zimmermann, } \\
\text { Wittenberg 1738. }\end{array}$ \\
\hline $\begin{array}{c}\text { Karl Andreas } \\
\text { Christiani }\end{array}$ & \multicolumn{1}{c|}{ (ns) } \\
\hline $\begin{array}{c}\text { Karl Heinrich Rappolt } \\
\text { Ludwig Thümmig, Institutiones philosophiae wolfianae, Renger, Frankfurt } \\
\text { 1725-1726. }\end{array}$ \\
\hline Buck & $\begin{array}{l}\text { Christian Wolff, Vernünftige Gedanken von Gott, der Welt und der Seele } \\
\text { des Menschen, auch allen Dingen überhaupt, Renger, Halle 1719. }\end{array}$ \\
\hline
\end{tabular}




\begin{tabular}{|c|c|}
\hline Professors & Summer Semester 1754 \\
\hline $\begin{array}{c}\text { Karl Andreas } \\
\text { Christiani }\end{array}$ & (ns), (cp) \\
\hline $\begin{array}{c}\text { Friedrich J ohann } \\
\text { Buck }\end{array}$ & (ns), (cp) \\
\hline
\end{tabular}

\begin{tabular}{|c|l|}
\hline Professors & \multicolumn{1}{|c|}{ Winter Semester 1754/1755 } \\
\hline J ohann David Kypke & $\begin{array}{l}\text { Friedrich Christian Baumeister, Institutiones metaphysicae, Zimmermann, } \\
\text { Wittenberg 1738. }\end{array}$ \\
\hline $\begin{array}{c}\text { Coelestin Christian } \\
\text { Flottwell }\end{array}$ & (ns) \\
\hline Georg David Kypke & (ns), (cp) \\
\hline
\end{tabular}

\begin{tabular}{|c|c|}
\hline Professors & Summer Semester 1755 \\
\hline $\begin{array}{l}\text { Karl Andreas } \\
\text { Christiani }\end{array}$ & $(\mathrm{ns}),(\mathrm{cp})$ \\
\hline $\begin{array}{l}\text { Coelestin Christian } \\
\text { Flottwell }\end{array}$ & (ns), (cp) \\
\hline Georg David Kypke & $\begin{array}{l}\text { Friedrich Christian Baumeister, Institutiones metaphysicae, Zimmermann, } \\
\text { Wittenberg } 1738 .\end{array}$ \\
\hline $\begin{array}{l}\text { Friedrich J ohann } \\
\text { Buck }\end{array}$ & (ns) \\
\hline
\end{tabular}

\begin{tabular}{|c|l|}
\hline Professors & \multicolumn{1}{|c|}{ Winter Semester 1755/1756 } \\
\hline J ohann David Kypke & $\begin{array}{l}\text { Friedrich Christian Baumeister, Institutiones metaphysicae, Zimmermann, } \\
\text { Wittenberg 1738. }\end{array}$ \\
\hline $\begin{array}{c}\text { Karl A ndreas } \\
\text { Christiani }\end{array}$ & (ns), (cp) \\
\hline $\begin{array}{c}\text { Friedrich J ohann } \\
\text { Buck }\end{array}$ & (ns), (cp) \\
\hline
\end{tabular}

\begin{tabular}{|c|l|}
\hline $\begin{array}{c}\text { Professors } \\
\text { Johann Friedrich } \\
\text { Werner }\end{array}$ & $\begin{array}{l}\text { Friedrich Christian Baumeister, Institutiones metaphysicae, Zimmermann, } \\
\text { Wittenberg 1738. }\end{array}$ \\
\hline $\begin{array}{c}\text { Coelestin Christian } \\
\text { Flottwell }\end{array}$ & (ns) \\
\hline Friedrich J ohann & (ns) \\
\hline
\end{tabular}




\begin{tabular}{|c|c|}
\hline Professors & \multicolumn{1}{|c|}{ Winter Semester 1756/1757 } \\
\hline J ohann David Kypke & $\begin{array}{l}\text { Friedrich Christian Baumeister, Institutiones metaphysicae, Zimmermann, } \\
\text { Wittenberg 1738. (p) }\end{array}$ \\
\hline $\begin{array}{c}\text { Coelestin Christian } \\
\text { Flottwell }\end{array}$ & (ns), (cp) \\
\hline $\begin{array}{c}\text { Friedrich J ohann } \\
\text { Buck }\end{array}$ & (ns) \\
\hline $\begin{array}{c}\text { Matthias Friedrich } \\
\text { Watson }\end{array}$ & Alexander G. Baumgarten, Metaphysica, Hemmerde, Halle 1739. (p) \\
\hline
\end{tabular}

\begin{tabular}{|c|c|}
\hline $\begin{array}{c}\text { Professors } \\
\text { Karl Andreas } \\
\text { Christiani }\end{array}$ & \multicolumn{1}{|c|}{ Summer Semester 1757 } \\
\hline $\begin{array}{c}\text { Coelestin Christian } \\
\text { Flottwell }\end{array}$ & (ns) \\
\hline $\begin{array}{c}\text { Friedrich J ohann } \\
\text { Buck }\end{array}$ & \multicolumn{1}{c|}{ (ns) } \\
\hline $\begin{array}{c}\text { Matthias Friedrich } \\
\text { Watson }\end{array}$ & $\begin{array}{l}\text { Christian Friedrich Ammon, Lineae primae eruditionis humanae, Reusner, } \\
\text { Königsberg 1737. }\end{array}$ \\
\hline Heinrich Oelmann & \\
\hline
\end{tabular}

\begin{tabular}{|c|l|}
\hline Professors & \multicolumn{1}{|c|}{ Winter Semester 1757/1758 } \\
\hline J ohann David Kypke & $\begin{array}{l}\text { Friedrich Christian Baumeister, Institutiones metaphysicae, Zimmermann, } \\
\text { Wittenberg 1738. (p) }\end{array}$ \\
\hline $\begin{array}{c}\text { Matthias Friedrich } \\
\text { Watson }\end{array}$ & $\begin{array}{l}\text { Friedrich Christian Baumeister, Institutiones metaphysicae, Zimmermann, } \\
\text { Wittenberg 1738. }\end{array}$ \\
\hline
\end{tabular}

\begin{tabular}{|c|l|}
\hline $\begin{array}{c}\text { Professors } \\
\text { Matthias Friedrich } \\
\text { Watson }\end{array}$ & \begin{tabular}{l}
\multicolumn{1}{|c|}{ Summer Semester 1758 } \\
Wittenberg 1738.
\end{tabular} \\
\hline
\end{tabular}

\begin{tabular}{|c|c|}
\hline Professors & Winter Semester 1758/1759 \\
\hline Karl Andreas Christiani & $(n s),(c p)$ \\
\hline $\begin{array}{l}\text { Coelestin Christian } \\
\text { Flottwell }\end{array}$ & (ns) \\
\hline $\begin{array}{l}\text { Matthias Friedrich } \\
\text { Watson }\end{array}$ & $\begin{array}{l}\text { Friedrich Christian Baumeister, Institutiones metaphysicae, Zimmermann, } \\
\text { Wittenberg 1738. (p) }\end{array}$ \\
\hline
\end{tabular}




\begin{tabular}{|c|c|}
\hline Professors & Summer Semester 1759 \\
\hline Karl Andreas & (ns), (cp) \\
Christiani & \\
\hline $\begin{array}{c}\text { Friedrich J ohann } \\
\text { Buck }\end{array}$ & (ns) \\
\hline
\end{tabular}

\begin{tabular}{|c|c|}
\hline Professors & Winter Semester 1759/1760 \\
\hline Karl Andreas & (ns), (cp) \\
Christiani & \\
\hline $\begin{array}{c}\text { Friedrich J ohann } \\
\text { Buck }\end{array}$ & (ns) \\
\hline
\end{tabular}

\begin{tabular}{|c|c|}
\hline Professors & Summer Semester 1760 \\
\hline Karl Andreas & (ns), (cp) \\
Christiani & \\
\hline $\begin{array}{c}\text { Friedrich J ohann } \\
\text { Buck }\end{array}$ & (ns) \\
\hline
\end{tabular}

\begin{tabular}{|c|c|}
\hline $\begin{array}{c}\text { Professors } \\
\text { Karl Andreas } \\
\text { Christiani }\end{array}$ & \multicolumn{1}{|c|}{ Winter Semester 1760/1761 } \\
\hline $\begin{array}{c}\text { Friedrich J ohann } \\
\text { Buck }\end{array}$ & Alexander G. Baumgarten, M etaphysica, Hemmerde, Halle 1739. \\
& $\begin{array}{l}\text { Christian August Crusius, Entwurf der nothwendigen Vernunft- } \\
\text { Wahrheiten, Gledtisch, Leipzig 1745. }\end{array}$ \\
\hline
\end{tabular}

\begin{tabular}{|c|c|}
\hline Professors & Summer Semester 1761 \\
\hline Karl Andreas & (ns), (cp) \\
Christiani & \\
\hline $\begin{array}{c}\text { Friedrich J ohann } \\
\text { Buck }\end{array}$ & (ns) \\
\hline
\end{tabular}

\begin{tabular}{|c|cccc|}
\hline Professors & \multicolumn{4}{|c|}{ Winter Semester 1761/1762 } \\
\hline $\begin{array}{c}\text { Karl Andreas } \\
\text { Christiani }\end{array}$ & \multicolumn{4}{|c|}{ (ns), (cp) } \\
\hline Friedrich J ohann & Christian & August & Crusius, Entwurf der nothwendigen Vernunft- \\
\hline
\end{tabular}




\begin{tabular}{|c|c|}
\hline Professors & Winter Semester 1762/1763 \\
\hline $\begin{array}{c}\text { Karl Andreas } \\
\text { Christiani }\end{array}$ & (ns), (cp) \\
\hline $\begin{array}{c}\text { Friedrich J ohann } \\
\text { Buck }\end{array}$ & (ns) \\
\hline
\end{tabular}

\begin{tabular}{|c|c|}
\hline Professors & Summer Semester 1763 \\
\hline Karl Andreas & (ns), (cp) \\
Christiani & (ns) \\
\hline $\begin{array}{c}\text { Friedrich J ohann } \\
\text { Buck }\end{array}$ & \\
\hline
\end{tabular}

\begin{tabular}{|c|c|}
\hline Professors & Winter Semester 1763/1764 \\
\hline Karl Andreas & (ns), (cp) \\
Christiani & (ns) \\
\hline $\begin{array}{c}\text { Friedrich J ohann } \\
\text { Buck }\end{array}$ & \\
\hline
\end{tabular}

\begin{tabular}{|c|c|}
\hline Professors & Summer Semester 1764 \\
\hline Karl Andreas & (ns), (cp) \\
Christiani & (ns) \\
\hline $\begin{array}{c}\text { Friedrich J ohann } \\
\text { Buck }\end{array}$ & \\
\hline
\end{tabular}

\begin{tabular}{|c|c|}
\hline Professors & Winter Semester 1764/1765 \\
\hline Karl Andreas & (ns), (cp) \\
Christiani & (ns) \\
\hline $\begin{array}{c}\text { Friedrich J ohann } \\
\text { Buck }\end{array}$ & \\
\hline
\end{tabular}

Summer Semester 1765

There were not lectures on metaphysics in this semester

\begin{tabular}{|c|c|}
\hline Professors & Winter Semester 1765/1766 \\
\hline Karl Andreas & (ns), (cp) \\
\hline
\end{tabular}




\begin{tabular}{|c|l|}
\hline Professors & \multicolumn{1}{|c|}{ Summer Semester 1767 } \\
\hline $\begin{array}{c}\text { Karl Andreas } \\
\text { Christiani }\end{array}$ & Friedrich Christian Baumeister, Institutiones metaphysicae, Zimmermann, \\
Wittenberg 1738. (p) \\
\hline $\begin{array}{c}\text { Friedrich J ohann } \\
\text { Buck }\end{array}$ & $\begin{array}{l}\text { Christian August Crusius, Entwurf der nothwendigen Vernunft- } \\
\text { Wahrheiten, Gledtisch, Leipzig 1745. (p) }\end{array}$ \\
\hline
\end{tabular}

\begin{tabular}{|c|l|}
\hline Professors & \multicolumn{1}{|c|}{ Winter Semester 1767/1768 } \\
\hline $\begin{array}{c}\text { Karl Andreas } \\
\text { Christiani }\end{array}$ & $\begin{array}{l}\text { Friedrich Christian Baumeister, Institutiones metaphysicae, Zimmermann, } \\
\text { Wittenberg 1738. (p) }\end{array}$ \\
\hline $\begin{array}{c}\text { Friedrich J ohann } \\
\text { Buck }\end{array}$ & $\begin{array}{l}\text { Christian August Crusius, Entwurf der nothwendigen Vernunft- } \\
\text { Wahrheiten, Gledtisch, Leipzig 1745. (p) }\end{array}$ \\
\hline
\end{tabular}

Summer Semester 1768

There were not lectures on metaphysics in this semester

\begin{tabular}{|c|l|}
\hline Professors & \multicolumn{3}{|c|}{ Winter Semester 1768/1769 } \\
\hline $\begin{array}{c}\text { Karl Andreas } \\
\text { Christiani }\end{array}$ & Friedrich Christian Baumeister, Institutiones metaphysicae, Zimmermann, \\
Wittenberg 1738. (p) & \\
\hline $\begin{array}{c}\text { Friedrich J ohann } \\
\text { Buck }\end{array}$ & Christian August Crusius, Entwurf der nothwendigen Vernunft- \\
\hline
\end{tabular}

\begin{tabular}{|c|c|}
\hline Professors & Summer Semester 1769 \\
\hline Karl Andreas & (cp) \\
Christiani & \\
\hline
\end{tabular}

\begin{tabular}{|c|ll|}
\hline Professors & \multicolumn{3}{|c|}{ Winter Semester 1769/1770 } \\
\hline $\begin{array}{c}\text { Karl Andreas } \\
\text { Christian }\end{array}$ & $\begin{array}{l}\text { Friedrich Christian Baumeister, Institutiones metaphysicae, Zimmermann, } \\
\text { Wittenberg 1738. (p) }\end{array}$ & \\
\hline $\begin{array}{c}\text { Friedrich J ohann } \\
\text { Buck }\end{array}$ & $\begin{array}{l}\text { Christian August Crusius, Entwurf der nothwendigen Vernunft- } \\
\text { Wahrheiten, Gledtisch, Leipzig 1745. (p) }\end{array}$ & \\
\hline
\end{tabular}




\section{The Nature of Metaphysics}

In order to understand the various metaphysical trends in Königsberg prior 1770 and to assess their impact upon Kant, it is essential a careful examination of the main characteristics of each metaphysics. Here I consider only the meaningful handbooks on metaphysics, which were usually taught in Königsberg's university and were adopted for two or more semesters or were extremely relevant in the period before 1724 .

A braham Calov was the prominent metaphysician of Königsberg of the $17^{\text {th }}$ century. In his book M etaphysica divina defines metaphysics as "scientia de ente" (CALOV, 1640, p. 4). Metaphysics is a part of a wider group of metaphysical sciences, which include: 1) gnostology; 2) noology; 3) metaphysics. Metaphysics is in turn divided in 1) general metaphysics or ontology and 2) special metaphysics. General metaphysics or ontology deals with the ens qua ens that in Calov corresponds to the knowable of gnostology (cognoscibile) (SGARBI, 2009b). Special metaphysics deals with ens in abstractione minori, particularly with immaterial substances and God. Calov was also the only philosopher of Königsberg to deal with transcendental philosophy before Kant. If the object of ontology was the ens as cognoscibile, the transcendental affections must refer to the ens, not as such, but as a knowable. According to Calov, the transcendental attributes did not denote a mere being, but a knowable, i.e., the transcendental attributes without which the knowable would not be the object of knowledge. It is evident that Calov's formulation is extremely close to the Kantian and importantly creates a shift of transcendental philosophy from the old metaphysics to the new transcendental logic. Calov's notion of transcendental, in opposition to Kant, how ever, instead of referring to the knowing subject, it expresses the relation between the constituent parts of the object. Nonetheless Calov's metaphysical works for its large and rapid dissemination made Königsberg a stronghold of A ristotelian-Scholastic metaphysics.

Christian Dreier's Sapientia sive philosophia prima differs remarkably from the other metaphysical projects of the time. In fact, instead of a new elaboration of metaphysical sciences, Dreier aims to restore a pure A ristotelian metaphysics against the Scholastic intrepretation. He comments therefore prevalently the A ristotelian writings and asserts that metaphysics deals with being qua being and it is first philosophy and the most important science (DREIER, 1644, p. 38).

The last important exponent of Königsberg's A ristotelian metaphysics in the $18^{\text {th }}$ century was Paul Rabe. Rabe's Aristotelianism does not focus particularly on metaphysics, which was also investigated most of the time in logical terms. Metaphysics is a part of theoretical sciences, which include 
1) ontology; 2) noology; 3) pneumatology. M etaphysics has God as a proper subject (RABE, 1703, p. 1205). Ontology deals with ens qua ens, i.e., the being considered in a universal way (RABE, 1703, p. 1205). Pneumatology studies immaterial substances (RABE, 1703, p. 1206). Noology investigates the first principles of being qua being and it is a necessary propaedeutic science to metaphysics (RABE, 1703, p. 1206). Also his introductive works on logic were ufficially adopted in the Collegium Fridericianum and at the Albertina and were very widespread up to third decades of $18^{\text {th }}$ century.

Franz A lbert A epinus is a typical figure of German Scholasticism, which aims to elaborate, in Suárez's wake, a neutral science, i.e. ontology, to investigate the being qua being as propaedeutic to theology, which deals with immaterial substances, God and their attributes. He was also a strong opponent of Wolffianism and his work was used in Königsberg during the epoch of transition from the conservative A ristotelian metaphysics to the new Wolffian philosophy (SPALDING, 1804, p. 4). In his book Introductio in philosophiam, Aepinus identifies metaphysics with transcendental philosophy, which was a synonymous of ontology. Metaphysics is defined "ontologia, seu doctrina de ente ejusque inferioribus atque affectionis" (AEPINUS, 1714, p. 2). M etaphysics is "scientia theoretica de ente ejusque inferioribus generalioribus, quatenus abstract sunt a materia sensibili realiter, et quidem permissive seu secundum indifferentiam" (AEPINUS, 1714, p. 5). Ontology is the science of "de ente eiusque generalibus inferioribus, quatenus de rebus materialibus et immaterialibus, finiti et infinits dici possunt" (AEPINUS, 1714, p. 5). Recently the Kant-Forschung has focused its interest on A epinus as Kant's source of the doctrine of transcendentals (TOM M ASI, 2003), as Norbert Hinske had suggested already in 1968 (HIN SKE, 1968, p. 84-85). In Königsberg A epinus's works were taught between 1724 to 1727 by Thomas Burckhard. In his works, however, A epinus uses the term "transcendental" as a synonymous with "transcendent", which is contrary to the meaning that occurs in Kant's writings and therefore he had not a real or at least determining influence upon the Kantian philosophy.

At the beginning of the $18^{\text {th }}$ century Eclecticism became a popular philosophical movement. Eclectic philosophy generally denies the importance of metaphysics, which has usually to be subordinated to theology that posseses the real truth. For this reason Pietism supports Eclectic philosophy and during the Pietists's domain in Königsberg, metaphysics was regularly taught in the faculty of theology instead of in the faculty of philosophy. Christian Thomasius's attitude against the AristotelianScholastic metaphysics is representative of the Eclectic movement. M etaphysics is "regina, sed inter illas disciplinas, quae circa falsa et erronea sunt occupatae, quae fatagunt circa commenta astutorum et toiosorum 
hominum destinendis ingeniis, ne ad solidam rerum cognitionem adspirent, quae adhuc a multis mordicus retinentur, inscitia meliorum, aut quia dediscere pudet, quae jam otiose didicerunt" (THOMASIUS, 1688, p. 198). Therefore the subject of metaphysics are subtleties and otiose things and its aim is not the truth, but decieving. According to Budde, A ristotelian metaphysics could be a science only considering the ens qua ens not as the ens in abstracto, as the Schulmetaphysik did, but as the Ens primum, the Substantia prima, which is God, i.e. to make of metaphysics in theology. However, there was in Königsberg a striking case in which Aristotle's metaphysics was used to legitimize Eclectic philosophy. In his Meditatio philosophica qua Aristotelem sapientissimum de veritate judicem, Rohde, commenting the passage of A ristotle's M etaphysica II.1993 a 30 - "the study of truth is in one sense difficult, in another easy. This is shown by the fact that whereas no one person can obtain an adequate grasp of it, we cannot all fail in the attempt" -, affirms that the fact that no one person can fail in the attempt to discover the truth supports the Eclectic position according to which all the opinions of the past philosophers on metaphysics were important (ROHDE, 1722, p. 4).

In opposition to Aristotelian and Eclectic metaphysics was Wolffian metaphysics. Wolff developed for the first time his metaphysics in the Vernünftige Gedanken von Gott, der Welt und der Seele des M enschen, auch allen Dingen überhaupt (=Deutsche M etaphysik), which was published in 1719. Wolff's metaphysics inaguarated early a new trend in Königsberg already from the summer semester 1720 . In spite of this early dissemination, it is remarkable that in Königsberg only the German works of Wolff were taught and not the Latin books, as for example the Ontologia. In the Deutsche Metaphysik, Wolff does not give a definition of metaphysics, which is, however, the science that studies 1 ) everything is from different points of view and 2) what makes sense of it, that is God. M etaphysics is divided in: 1) ontology; 2) psychology; 3) cosmology; 4) theology.

Ludwig Thümmig's Institutiones philosophiae wolfianae disseminated for the first time in Königsberg the Wolffian metaphysics in Latin. This book was very widespread in Königsberg, especially among the junior professors of the faculty, and was usual taught for the cursus philosophicus instead of A ristotelian and Eclectic handbooks. Thümmig defines metaphysics as "scientiam entis et mundi in genere rerumque immaterialium" (THÜM MIG, 1725-1726, p. 39). Metaphysics is divided in: 1) ontology; 2) psychology or peneumatology; 3) natural theology; 4) general cosmology. Ontology is first philosophy and is "scientia entis in genere" (THÜM MIG, 1725-1726, p. 39). General cosmology is "scientia mundi in genere" (THÜM M IG, 1725-1726, p. 
71). Psychology is scientia animae, seu ea pas metaphysicae, quae de anima agit" (THÜM M IG , 1725-1726, p. 115). Subjects of metaphysics are therefore the being qua being, the soul, the world, and God.

Also Friedrich Christian Baumeister's Institutiones metaphysicae were extremely faithful to Wolff's metaphysics. Baumeister states that "scientia autem universalium, sive disciplina illa, in qua notiones, a rebus indivdui abstractae, et iisdem communes, distincte explicantur, generali nomine dicitur M etaphysica" (BAUM EISTER, 1738, p. 3). M etaphysics is divided in 1) ontology; 2) cosmology; 3) psychology; 4) natural theology. Ontology or first philosophy deals with "prima et comunissima omnis cognitionis hamanae principia explicantur, item notione sgeneralissimae et universaliores enodantur distincte et positiones, ob universalitatem suam" (BAUMEISTER, 1738, p. 5). Cosmology deals with "in qua de universo in genere agitur, et notionibus generalibus et abstractis de mundo explicatis, non nisi talia de mundo demosntratur quae mundo, ut enti composito et mutabili tribuuntur" (BAUMEISTER, 1738, p. 5-6). Psychology deals with the soul in two sense: "1) ea traditi, quae per experientiam de anima nobis innotescunt; 2) ea demonstrat, quae de anima, ope ratiociniorum, ex experientiis, rite stabilitis, deductorum nosse nobis datus est" (BAUM EISTER, 1738, p. 6). Natural theology deals with "Deo, eius existentia, attributis et operibus" (BAUMEISTER, 1738, p. 6).

An eclectic philosopher, who was influenced by Wolffian metaphysics, was also J ohann Christoph Gottsched. The first volume of his Erste Gründe der gesammten Weltweisheit is completely devoted to the theoretical philosophy. Thereoretical philosophy or metaphysics is divided in logic (Vernunftlehre), ontology (Grundlehre), cosmology (Weltweisheit von der Welt), peneumatology (Geisterlehre) and natural theology as in all the other Wolffian companions.

It is notew orthy that all the Wolffian manuals that were usually taught at the Albertina did not deal with the doctrine of transcendentals, which became a central topic only in Wolff's Ontologia, which, as I have just pointed out, was not a matter of teaching.

A nother important metaphysical book, which is often usually understood in the light of the Wolffian metaphysicis, was A lexander Gottlieb Baumgarten's M etaphysica. Although Baumgarten's metaphysics seems to be very similar to Thümmig and Baumeister's handbooks, and for its external subdivision could be also included in the Wolffian school, I want firmly to defend with Mario Casula the thesis that it was not a Wolffian (CASULA, 1973, p. 7), in fact his doctrines on being, essence and transcendentals were very different from those of the Wolffian tradition. The book is very famous 
because it was Kant's favourite metaphysical treatise and was his source of many metaphysical topics as for example the doctrine of the transcedentals. Metaphysics is "scientia primorum in humana cognitione principiorum" (BAUM GARTEN, 1739, p. 1). Metaphysics is divided in 1) ontology; 2) cosmology; 3) psychology; 4) natural theology. Ontology deals with the general predicates of being; cosmology deals with the general predicates of the world either "ex experientia proprius" (cosmologia empirica) or "ex notione mundi" (cosmologia rationalis); psychology deals with the general predicates of the soul either "ex experientia proprius" (pyschologia empirica) or "ex notione animae" (psychologia rationalis); natural theology deals with the concept of God and his operations.

A mong independent or eclectic positions there is J ohann Georg Walch with his Introductio in Philosophiam. According to Walch, metaphysics is "disciplinam philosophicam, quae de notionibus rerum communibus generatim, et speciatim de substantia nobilisssima; sive de Deo agit" (WALCH, 1730, p. 180). M etaphysics is divided in three parts. The first part deals with the being (ente) that is also called ontology, the second part with created substances, and the third part with God (WALCH, 1730, p. 181). According to the divison of metaphysics, three are the proper subjects: 1 ) ens in general (WALCH, 1730, p. 182); 2) ens according to their vim operandi, i.e., as principii and principiati, as causa and effectu (WALCH, 1730, p. 189) ; 3) ens per se subsistens, which is God (WALCH, 1730, p. 196).

The most important opponent to Wolff's metaphysics was Christian A ugust Crusius with his Entwurf der nothwendigen Vernunft-Wahrheiten, which Kant read carefully early in the Sixties (KGS, X, p. 32). M etaphysics, according to Crusius, is the science of the necessary truth of reason and it is divided in 1) ontology; 2) natural theology; 3) cosmology; 4) pneumatology. Ontology deals with the being of things and their properties that are knowable and explainable a priori (CRUSIUS, 1766, p. 7). The proper subject of natural theology is God as the necessary absolute substance (CRUSIUS, 1766, p. 7). Cosmology studies the necessary laws of the physical world while pneumatology the necessary laws of the spiritual world (CRUSIUS, 1766, p. 7-8).

\section{Conclusions}

From an accurate examination of the teachings of metaphysics from 1703 to 1770 it is possible to say that on one hand the Albertina was a very conservative university, but on the other hand it was extremely receptive. It was conservative because the A ristotelian tradition had long lasting influence 
for at least the first four decades of the $18^{\text {th }}$ century. It was receptive because the professors lectured on new releases, as in the case of Wolff, Gentzken, Thümmig, and Crusius's handbooks. The readiness with which some Königsberg professors favorably reacted to the new publications is, according to Giorgio Tonelli, "a symptom, in the first place, that the Königsberg intellectuals positively responded to intellectually alert, and, in the second place, that some tensions were present (mainly involving the junior faculty members), which prompted some instructors to an almost immediate acceptation of the new creed as a group ideology" (TONELLI, 1975, p. 132). Tonelli adds, however, that "the full professors of the philosophical faculty were impervious to the new trend" (TONELLI, 1975, p. 132). This could mean not only that the professors were open to new trends, but also that the handbooks were not so important for the teaching if they were changed so frequently and that the lectures were based prevalently on dictate on a common standard knowledge, which was releated to the A ristotelianScholastic tradition that domintated Königsberg's university for over twohundred years.

Looking at the professors, it is possible to say that until the second decade of the $18^{\text {th }}$ century they were Aristotelians. Following, besides A ristotelianism, for a short period of time Eclecticism and Wolffianism coexisted. Eclecticism, which was supported by Pietists, took the leadership from the second half of the Twenties to the second half of the Forties. After that there was a important return to Wolffianism.

This chronology confirms Tonelli's argumentations on the long survival of A ristotelianism in Königsberg at least up to 1725 with the leadership of J ohann J akob Quandt (TONELLI, 1975, p. 127-128). Between 1715 and 1740, A ristotelian, Eclectic, and Wolffian philosophies fought to extend their hegemony in the faculty of philosophy. Between 1715 and 1724 in Königsberg there was born a unusual coalition between Pietists and Wolffians against the A ristotelian conservatism, which culminated in 1725 when Wilhelm I nominated the pietist Gottfried Friedrich Rogall as full professor at the faculty of theology. In 1726 Rogall with Heinrich Lysius introduced a university reform in conformity with Pietism, against Wolffianism, which was substantially banished until 1740 when Friedrich II became king (TONELLI, 1975, p. 129).

Wolffianism was never a dominant metaphysical movement in Königsberg's university, in fact, no professor could declare himself truly a Wolffian scholar and no Wolffians became full professor in the chair of logic and metaphysics (POZZO, 2008, p. 182). M oreover the banishment of Wolffian philosophy by Pietists in a pietist university had to have an impact in the teaching. 
In addition two of the four professors who were very close to Kant, Kypke and Teske, defined themselves as Aristotelians, while, the other two professors, Marquardt and Knutzen, were very well educated in A ristotelian philosophy, even if they addressed their interests toward more eclectic positions.

In the light of those elements, it is not surprising that during his university years Kant received an eclectic education and broad knowledge of different metaphysical positions. The various metaphysical attempts of the pre-critical period have to be understood beginning from the different stimuli to which Kant was submitted and that he had carefully to consider before taking a serious position towards them. The study of Kant's early writings can not be separated from an investigation of their cultural context that enriches their value and justify the various Kantian perspectives.

SGARBI, Marco. A metafísica em königsberg antes de kant [1703-1770]. Trans/Form/Ação, (São Paulo); v.33(1), 2010, p.31-64.

RE SU M 0: 0 presente trabalho, valendo-se da metodologia da história das idéias, visa reconstruir o amplo espectro de doutrinas metafísicas às quais Kant poderia ter acesso durante os anos de formação de sua filosofia. A primeira parte trata do ensino de metafísica em Königsberg, de 1703 a 1770. A segunda parte examina as principais características da metafísica com base nos diversos manuais em uso na A lbertina, a fim de que se tenha um panorama exaustivo de todas as posições então objeto de ensino.

PALAVRAS-CHAVE: Metafísica. Ecletismo. Wolffianismo. Aristotelismo. Kant. Königsberg. Quellengeschichte.

\section{Bibliography}

AEPINUS, F. A. Introductio in philosophiam. Rostock: Wilde, 1714.

BAUM EISTER, F. C. Institutiones metaphysicae. Wittenberg: Zimmermann, 1738.

BAUM GARTEN, A. G. M etaphysica. Halle: Hemmerde, 1739.

CALOV, A. Metaphysica divina. Rostock: Hallervord, 1640.

CASULA, M. La metafisica di A.G. Baumgarten. Milano: Mursia, 1973.

CRUSIUS, C. A. Entwurf der nothwendigen Vernunft-Wahrheiten. Leipzig: Gledtisch, 1766. 
DREIER, C. Sapientia sive philosophia prima. Königsberg: Hendel, 1644.

ERDMANN, E. Martin Knutzen und seine Zeit. Leipzig: Voss, 1876.

GEORGI, H. Philosophia propaedeutica sive philosophiae fundamenta praerequisita ad ductum et methodum cursus philosophicis professoris Raben. Königsberg: Zaencker, 1716.

GOTTSCHED, J. C. Erste Gründe der gesammten Weltweisheit. Leipzig: Breitkopf, 1733-1734.

HINSKE, N. Die historischen Vorlagen der kantischen Transzendentalphilosophie. A rchiv für Begriffsgeschichte, v. 12, p. 86-113, 1968.

. Che cosa significa e a qual fine si pratica la storia delle fonti? Alcune osservazioni di storia delle fonti sulla antinomia kantiana della libertà. Studi Kantiani, v. 19, p. 113-120, 2006.

KANT'S gesammelte Schriften. Berlin: De Gruyter, 1900-

PISANSKI, G. C. Entwurf einer preußischen Literärgeschichte in vier Büchern. Königsberg: Hartung, 1886.

POZZO, R. Catalogus praelectionum academiae regiomontanae 1719-1804. Studi Kantiani, v. 4, p. 163-187, 1991.

. Logic and metaphysics in german philosophy from Melanchthon to Hegel. In: SWEET, W. (Ed.). A pproaches to metaphysics. Dordrecht: Kluwer, 2004. p. 61-74.

. Aristotelismus und Eklektismus in Königsberg (1648-1740). In: M ARTI, H.; KOM OROWSKI, M. (Ed.). Die Universität Königsberg in der frühen Neuzeit. Köln-Weimar-Wien: Böhlau, 2008. p. 172-185.

RABE, P. Cursus philosophicus, seu Compendium praecipuarum scientiarum philosophicarum, Dialecticae nempe, Analyticae, Politicae, sub qua comprehenditur Ethica, Physicae atque M etaphysicae, ex evidentioribus rectae rationis principiis deductum, methodo scientifica adornatum, et brevi atque perspicuo stylo concinnatum, in gratiam non solum Philosophiae cultorum ex professo, sed et imprimis eorum, qui tantum ex ea modo haurire desiderant, quantum sibi in superioribus Facultatibus usui esse potest in Theologia nempe, J urisprudentia et Medicina. Königsberg: Boye, 1703.

ROHDE, J. J. Meditatione philosophica qua Aristotelica sapientissimus de veritate. Königsberg: Reusner, 1722.

SGA RBI, M. Kant, Rabe e la logica aristotelica. Rivista di Storia della filosofia, v. 2, p. 289-313, 2009a.

. Unus, Verus, Bonus et Calovius. L'oggetto della metafisica secondo A braham Calov. Medioevo, v. 34. p. 381-398, 2009b. 
SPALDING, J. J . Lebensbeschreibung vom inm selbst aufgesetzt. Halle: Waisenhaus, 1804.

THOM ASIUS, C. Introductio in philosophiam aulicam. Leipzig: Thomasius, 1688.

THÜMMIG, L. Institutiones philosophiae wolfianae. Frankfurt: Renger, 17251726.

TOMMASI, F. V. Kant di fronte alla tradizione del "trascendentale": stato della ricerca e prospettive alla luce di un nuovo particolare. Studi Kantiani, v. 16, p. 53-66, 2003.

TONELLI, G. Conditions in Königsberg and the Making of Kant's Philosophy. In: BUCHER, A . J .; DRÜE, H.; SEEBOHM , T. M . (Ed.). Bewusst-sein. Bonn: Bouvier, 1975. p. 126-144.

WALCH, J. G. Introductio in philosophiam. Leipzig: Gleditsch, 1730. 